\section{SEEING IS BELIEVING}

New to GC UK's product range is Tri Plaque ID Gel. As the name suggests Tri Plaque ID Gel allows you and your patient to identify areas of plaque in three easy steps.

GC's Tri Plaque ID Gel quickly identifies new, mature and acid producing biofilms. This unique gel also highlights exactly where the bacteria are most active by disclosing the acidic $\mathrm{pH}$. This additional information will be a great

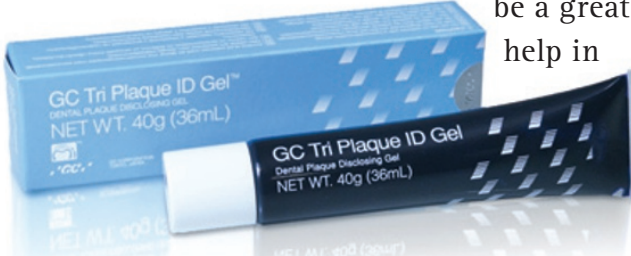

your daily practice to motivate your patients to improve their oral hygiene. After all, people often need to see something before they believe it.

The gel is colour coded for easy identification:

- Blue/purple - old plaque (more than 48 hours)

- Red/pink - newly formed plaque

- Light blue - high risk plaque.

Tri Plaque ID Gel encourages patients to be more precise with their tooth brushing technique. Once diagnosis is complete simply brush the teeth to clean them.

For further information please contact GC UK on 01908218999.

\title{
A VARIETY OF COSMETIC SURGERY APPLICATIONS
}

When practices buy a SiroLaser Advance from Sident Dental Systems, they can use it to whiten teeth using its bleaching applications. They can also use it to enhance aesthetic results by improving impression taking procedures by facilitating quicker exposure of crown margins and eliminating bleeding due to its strong haemostatic effect, creating more predictable results and reducing the number of post-operative complaints.

SIROLaser Advance can be used for soft tissue surgery in a variety of cosmetic dentistry applications as well as for disinfection in endodontic treatment, crown lengthening, treating periodontal pockets, resolving peri-implantitis, and bleaching applications. In surgi- cal applications it offers high precision tissue removal with minimal trauma to surrounding tissues, minimised bleeding for clearer visibility, protection against post-operative infection, minimised scar formation, an enhanced healing response and virtually no post-operative pain.

The easy-to-use touch screen menu makes it is very simple to set the treatment parameters because the most frequently used clinical applications have already been preset. To activate the SIROLaser Advance the operator can either use the wireless foot control or the finger switch found on the ergonomically shaped hand piece.

For further information call Sident Dental Systems on 01932582900 or email info@sident.co.uk.

\section{SEVEN SPECIAL BENEFITS FOR SENSITIVE TEETH}

Sensodyne Complete Protection from GlaxoSmithKline Consumer Healthcare (GSK) has seven specially-designed benefits and is targeted to sufferers of tooth sensitivity. Sensodyne Complete Protection is available in Sensodyne Complete Protection and Sensodyne Complete Protection Extra Fresh.

Sensodyne Complete Protection creates a mineral layer over sensitive areas to shield you from the pain of sensitive teeth; strengthens and rehardens enamel; gives a clean feeling; helps maintain healthy gums; freshens breath; helps control plaque; and helps maintain natural whiteness.

In addition, Sensodyne is launching two new Complete Protection toothbrushes, soft and medium. The brushes are available in four different colour options and feature a small angled head. With soft outer bristles and twisting inner bristles, the brushes are speciallydesigned for people with sensitive teeth and provide added benefits of plaque removal, enamel and gum cleaning.

All new products are available now. www.gsk-dentalprofessionals.co.uk. 